\title{
Perspective
}

\section{New perspectives on vascular wall signaling: role of perivascular adipocytes and fibroblasts}

\author{
Chiu-Yin KWAN ${ }^{1, *}$, Wen-Tsong HSIEH ${ }^{1}$, Peter Nim-Hin TO ${ }^{1}$, Hui-Di WANG ${ }^{2}$ \\ ${ }^{1}$ Graduate Institute of Basic Medical Sciences, China Medical University and Hospitals, Taichung, Taiwan, China; ${ }^{2}$ Department of \\ Community Health Sciences, Brock University, St Catharines, ON, Canada
}

\begin{abstract}
This communication represents personal perspectives of recent development in the newly evolved areas in vascular signaling mechanisms at the anatomical level of vascular walls from outside in, that is, from perivascular adventitial side to effectuate the control of vascular reactivity. Since half a century ago, the focus of interest in vascular biology has been confined primarily to the study of the excitation-contraction coupling of vascular smooth muscle (VSM) as well as neuroeffector mechanisms. During the past 3 decades, considerable advancement in the understanding of vascular signaling has been made via the discovery of endotheliumderived relaxation factors (EDRF), endothelium-derived hyperpolarizing factors (EDHF) and endothelium-derived contracting factors (EDCF). The discovery of nitric oxide (NO) as a major cellular messenger has also helped open up another huge area of research in oxidative stress and vascular diseases. In the past decade, concepts on vascular wall signaling have been extended from vascular endothelial cells and then translated to the other seemingly inert cellular components, such as perivascular adipocytes and adventitial fibroblasts. Growing body of evidences show that these cellularities contribute to both functional as well as structural integrity in vasculature with significant pathophysiological implications.
\end{abstract}

Keywords: vascular walls; perivascular adipocyte; vascular adventitial fibroblasts; smooth muscle cells; endothelium; vascular reactivity

Acta Pharmacologica Sinica (2010) 31: 1022-1025; doi: 10.1038/aps.2010.148; published online 16 Aug 2010

Blood vessels represent a complex organ with an anatomically diversified system of various functionalities. For a long while, the final common pathway in the studies of vascular function has converged within the medial smooth muscle layer, where the vascular reactivity (the active force generation) and the extracellular matrixes (elastin sheets and collagen fibers providing the passive recoil force) represent the components in "myogenic control" of the vascular reactivity in response to physical stress and stretch. There have been two very common and contemporary ways of studying the control of vascular tone across the vessel walls. One has been at the level of "humoral control" that deals with the control of vascular reactivity by circulating hormones or other smaller molecules, which can be peptides, electrolytes, environmental toxins or non-peptide hormones. The other has been at the level of "neural control". As most blood vessels are innervated from the outer adventitial site, the "neurogenic control" of the vascular reactivity extends itself into various pharmacological domains including pre- and post-junction receptors and post-

\footnotetext{
* To whom correspondence should be addressed.

E-mail kwancy@mail.cmu.edu.tw

Received 2010-06-04 Accepted 2010-07-27
}

receptor signaling mechanisms.

\section{Signaling across vascular wall has come a long way: from inside out to outside in}

However, the excitement of studying signaling mechanisms in the vascular walls came about when the vascular endothelium at the intimal layer is no longer regarded as an inert one-cell layer insolating between vascular smooth muscle layer and the circulating humoral space. For more than two recent decades, the role of endothelium, the innermost layer of the vessel wall in the fine regulation of vascular tone has been well established with the discovery of EDRF, EDHF, and EDCF. The discovery of NO leading to its honoring with a Nobel Prize in medicine in the 90s marked the undeniable importance of the scientific discoveries in contemporary vascular biology and pushed the horizon into a newly developed dimension, ie, the role of reactive oxygen species (ROS) and anti-oxidative therapeutic approach in the treatment of vascular diseases, which may be related to the vascular complications in the inflammatory and aging process.

Considerable interests have now turned to the cellular components situated at perivascular site of the vascular wall. Indeed, other than the neuronal network, the external adven- 
titial site in most blood vessels also has substantial areas covered by adipocytes and fibroblasts next to the smooth muscle layer. Like vascular endothelium, the cellularity embedded in the vascular adventitia was initially thought to be relatively static and passive and offer only structurally supportive and anatomically protective roles. Recent evidence suggests that this has no longer been the case; both vascular adipocytes and fibroblasts have been shown to be actively involved in the control of vascular smooth muscle reactivity, and perhaps vascular growth. This aspect, now referred to as "paracrine control" of vascular reactivity, would be of particular contemporary interest in view of the role of metabolic syndrome and obesity in cardiovascular complications, such as hypertension and diabetes. However, despite novel discoveries reported in very recent years by several laboratories, whether and how these components are interacting to achieve an integrated control of vascular tone remain as a newly opened area of research in vascular biology.

\section{Perivascular adipocytes: diffusible factors and control of vascular reactivity}

It is now well established that obesity is associated with a state of chronic low grade inflammation involving the production of pro- and anti-inflammatory cytokines by white adipocytes, including those surrounding the vasculature ${ }^{[1,2]}$. The perivascular adipose tissue (PVAT) hitherto considered a passive structural support for blood vessels is now known to play a role in vascular tissue homeostasis and, therefore, blood pressure control ${ }^{[3,4]}$. Soltis and Cassis ${ }^{[5]}$ were the first to show that PVAT decreased the contractile sensitivity to noradrenaline in rat aorta, but had no effect on $\mathrm{KCl}$ and phenylephrine-induced contraction. Others have observed the inhibitory effect of PVAT on several pro-contractile agonists, including phenylephrine and serotonin ${ }^{[6]}$. More strikingly, the contraction to angiotension II was totally inhibited, an observation not being shared by other investigators. The interesting aspect was that the effect of PVAT could be demonstrated in a cascade system to show that diffusible substances from the fat tissue may be responsible for the observed inhibitory effects. Gao et al ${ }^{[4]}$ reported a dual mechanism for the anti-contractile effects, one that involves an endothelium-dependent relaxation via $\mathrm{NO}$ release and subsequent $\mathrm{K}_{\mathrm{Ca}}$ channel activation, and the other involving an endothelium-independent mechanism via $\mathrm{H}_{2} \mathrm{O}_{2}$ and subsequent release of soluble guanylate cyclase ${ }^{[7]}$. More recently, the adipocyte-derived relaxation factor (ADRF)-induced endothelium-dependent relaxation via NO release has been identified to be Ang-(1-7) ${ }^{[8]}$. Nonetheless, the physiological significance of finding in such in vitro study in the interaction between ADRF and EDRF from across the entire thickness of the vessels wall in vivo remains vague at large. Akin to EDRF, ADRF is reported to attenuate vasoconstriction to a wide spectrum of various agonists. Nonetheless, the results varied considerably as reported from different laboratories. Also, few reports have convincingly established the relationship between the amount of the perivascular fat tissues ${ }^{[9]}$, the incubation time and the magnitude of the functional alterations, especially in the cascade system. While the $\mathrm{K}_{\mathrm{ATP}}$ potassium channel was reported to mediate the relaxant effect of ADRF in aortic tissues ${ }^{[7]}$, the voltage-operated potassium channel was implicated in the mesenteric artery indicating possible regional differences in the modulation of PVAT function or the possibility of various types of $\mathrm{ADRF}^{[9]}$. ADCF, which is reported to include superoxide, exerts its contractile effect only upon electrical stimulation ${ }^{[10]}$, thus suggesting an interaction between perivascular nerve and fat tissues. The physiological significance remains unclear. However, Lee et $\mathrm{al}^{[11]}$ reported that the presence of PVAT potentiated $\mathrm{KCl}$ induced contraction in mesenteric arteries in Wistar rats. While the above findings clearly suggest some evidence for the "paracrine" role of PVAT ${ }^{[12]}$, a more vigor experimental approach is still necessary to unequivocally define the role of adipocyte-derived factors. The predominant finding of the inhibitory effect of ADRF on the vascular reactivity appears to argue against the exaggerated vascular reactivity or reduced endothelium-dependent relaxation commonly found to be linked with obesity-associated hypertension or metabolic syndrome ${ }^{[13]}$. It is worthwhile to mention that periaortic fat tissue has been found to stimulate vascular smooth muscle cells proliferation, a structural characteristics consistent with vascular changes associated with aging and obesity ${ }^{[14]}$. The stimulating or contractile effect of PVAT has so far been underplayed and not been receiving adequate investigation.

\section{Perivascular fibroblasts: source of ROS and regulation of vascular matrix}

Adventitial fibroblasts have been well known to produce copious extracellular matrix proteins, particularly collagen and elastin, for structural support of the vessel wall, but excessive deposition of vascular matrix proteins, especially collagen, as in systemic hypertension, occurs throughout the vessel with initial build-up taking place in the adventitial region of the vessel walls. Increased production of collagen has been demonstrated in adventitial fibroblasts due to stimulation with several mitogens, including Ang $\mathrm{II}^{[15]}$, an important cardiovascular regulator.

ET-1 as another major contributor in many pathological conditions, such as hypertension and atherosclerosis, can be expressed in adventitial. Recently, it has been reported that Ang II stimulation evokes expression of ET-1 in adventitial fibroblasts contributes to type I procollagen expression through activation of $\mathrm{ET}_{\mathrm{A}}$ receptors, suggesting a functional role for adventitial ET-1 release in the regulation of the extracellular matrix ${ }^{[15]}$. Expression of ET-1 in adventitial fibroblasts is mediated by $\mathrm{O}_{2}{ }^{-[16]}$, consistent with the findings in many different cell types ${ }^{[17]}$, including $\operatorname{VSM}^{[18,19]}$. However, the mechanisms of oxidative stress regulating ET-1 production are still unclear.

Adventitial fibroblasts are involved in arterial repair ${ }^{[20,21]}$ and serve as one of the major sources of vascular ROS production $^{[22-26]}$. Nicotinamide adenine dinucleotide phosphateoxidase (NADPH oxidase) has been well know as a key ROS 
generating enzyme in vasculature. Reduction in adventitial NADPH oxidase-derived ROS or overexpression of antioxidative enzymes reduces medial ROS levels and medial hypertrophy ${ }^{[20]}$, adventitial fibroblast migration in vitro ${ }^{[27]}$, and neointimal formation in a cuff-injured artery model ${ }^{[28]}$.

Evidence also suggests that the vascular adventitia may play an important role in vasomotor responses. The ability of the adventitia to respond to vasoactive peptides has been discovered recently in human tissue-engineered blood vessels ${ }^{[29]}$. On the other hand, the adventitia has been demonstrated to be a richer source of NO than the media, and that the adventitiaderived $\mathrm{NO}$ is able to activate guanylyl cyclase and vasodilatation within the media of the rat aorta ${ }^{[30,31]}$.

\section{Conclusion and future perspectives}

It has becoming clear that perivascular adipocytes and fibroblasts in the adventitial layer should no longer be considered a passive or static component of the vascular wall. They in fact represent active cellular entities to exert important paracrine actions and maintain the integral functions of vasculature. Adventitia is also a key source of vascular ROS attesting to its metabolically active signaling role via the regulation of oxido-reductive mechanisms. Thus, it provides a strategically unique adaptation in response to pathological conditions, such as injury, hypoxia and pulmonary hypertension, resulting in mediation of vascular remodeling, repair and extracellular matrix deposition. It may also play a functional role in regulating vascular tone. While the putative relaxant effects of perivascular adipocytes are of academic interest, some of the published evidence is still subject to methodological questions, physiological interpretation, especially with respect to its clinical significance in vascular dysfunction associated with obesity.

Recently, it has been reported that adventitial fibroblasts can produce ET-1 following Ang II treatment, which in turn mediates collagen synthesis in adventitial fibroblasts. These findings have important implications in disease state such as hypertension or diabetes which is associated with compromised function and structural remodeling of the vasculature which often accompanied the development of obesity. Despite the significant progresses that have been made, many important questions raised above regarding the role of adventitial function remain unanswered and require further research to determine its exact role in physiological and pathological conditions.

\section{References}

1 Hotamisligil GS. Inflammation and metabolic disorders. Nature 2006; 444: 875-80.

2 Lyon CJ, Law RE, Hsueh WA. Minireview: adiposity, inflammation, and atherogenesis. Endocrinology 2003; 144: 2195-200.

3 Fernandez-Alfonso MS. Regulation of vascular tone: the fat connection. Hypertension 2004; 44: 255-6.

4 Gao YJ. Dual modulation of vascular function by perivascular adipose tissue and its potential correlation with adiposity/ lipoatrophy-related vascular function. Curr Pharm Des 2007; 13: 2185-92.

5 Soltis EE, Cassis LA. Influence of perivascular adipose tissue on rat aortic muscle reasponsiveness. Clin Exp Hypertens A 1991; 13: 277-96.

6 Lohn M, Dubrovska G, Lauterbach B, Luft FC, Gollasch M, Sharma AM. Periadventitial fat releases a vascular relaxing factor. FASEB J 2002; 16: 1057-63.

7 Gao YJ, Lu C, Su LY, Sharma AM, Lee RMKW. Modulation of vascular function by perivascular adipose tissue: the role of endothelium and hydrogen peroxide. Br J Pharmacol 2007; 151: 323-31.

8 Lee RM, Lu C, Su LY, Gao YJ. Endothelium-dependent relaxation factor released by pervascular adipose tissue. J Hypertens 2009; 27: 782-90.

9 Verlohren S, Dudrovska G, Tsang SY, Essine K, Luft FC, Huang Y, Gollasch M. Visceral periadventitial adipose tissue regulates arterial tone of mesenteric arteries. Hypertension 2004; 44: 271-6.

10 Gao YJ, Takamori K, Su LY, An WS, Lu C, Sharma AM, et al. Perivascular adipose tissue promotes vasoconstriction: the told of superoxide anion. Cardiovasc Res 2006; 71: 363-73.

11 Lee RM, Ding L, Lu C, Su LY, Gao YL. Alteration of perivascular adipose tissue function in angiotensin II-induced hypertension. Can J Physiol Pharmacol 2009; 87:944-53.

12 Gollasch M, Dubrovska G. Pararcrine role for periadventitial adipose tissues in the regulation of arterial tone. Trends Pharmacol Sci 2004; 25:647-53.

13 Stapleton PA, James ME, Goodwill AG, Frisbee JC. Obesity and vascular dysfunction. Pathophysiology 2008; 15:79-89.

14 Baradier C, Montani JP, Yang Z. Mature adipocytes and perivascular adipose tissue stimulate vascular smooth muscle cell proliferation: effects of aging and obesity. Am J Physiol Heart Circ Physiol 2005; 289: $\mathrm{H} 1807-13$.

15 An SJ, Boyd R, Wang Y, Qiu X, Wang HD. Endothelin-1 expression in vascular adventitial fibroblasts. Am J Physiol Heart Circ Physiol, 2006; 290: H70O-H708.

16 An SJ, Boyd R, Zhu M, Chapman A, Pimentel DR, Wang HD. NADPH oxidase mediates angiotensin II-induced endothelin-1 expression in vascular adventitial fibroblasts. Cardiovasc Res 2007; 75: 702-9.

17 Wand HD, Rätsep MT, Chapman A, Boyd R. Aventitial fibroblasts in vascular structure and function: the role of oxidative stress and beyond. Can J Physiol Pharmacol 2010; 88: 177-86.

18 Kaehler J, Sill B, Koester R, Mittmann C, Orzechowski HD, Muenzel $\mathrm{T}$, et al. Endothelin-1 mRNA and protein in vascular wall cells is increased by reactive oxygen species. Clin Sci (Lond) 2002; 103 (Suppl 48): $176 S-178 S$.

19 Ruef J, Moser M, Kubler W, Bode C. Induction of endothelin-1 expression by oxidative stress in vascular smooth muscle cells. Cardiovasc Pathol 2001; 10: 311-5.

20 Liu J, Ormsby A, Oja-Tebbe N, Pagano PJ. Gene transfer of NAD(P)H oxidase inhibitor to the vascular adventitia attenuates medial smooth muscle hypertrophy. Circ Res 2004; 95: 587-94.

21 Shi Y, Pieniek M, Fard A, O'Brien J, Mannion JD, Zalewski A. Adventitial remodeling after coronary arterial injury. Circulation 1996; 93: 3408.

22 Ardanaz N, Pagano PJ. Hydrogen peroxide as a paracrine vascular mediator: regulation and signaling leading to dysfunction. Exp Biol Med (Maywood) 2006; 231: 237-51.

23 Wang HD, Pagano PJ, Du Y, Cayatte AJ, Quinn MT, Brecher P, et al. Superoxide anion from the adventitia of the rat thoracic aorta inactivates nitric oxide. Circ Res 1998; 82: 810-8.

24 Wang HD, Hope S, Du Y, Quinn, MT, Cayatte AJ, Pagano PJ, et al. Paracrine role of adventitial superoxide anion in mediating spontaneous tone of the isolated rat aorta in angiotensin II-induced hypertension. Hypertension 1999 ; 33: 1225-32. 
25 Wang HD, Xu S, Johns DG, Du Y, Quinn MT, Cayatte AJ, et al. Role of NADPH oxidase in the vascular hypertrophic and oxidative stress response to angiotensin II in mice. Circ Res 2001; 88: 947-53.

26 Wang HD, Johns DG, Xu S, Cohen RA. Role of superoxide anion in regulating pressor and vascular hypertrophic response to angiotensin II. Am J Physiol Heart Circ Physiol 2002; 282: H1697-H1702.

27 Haurani MJ, Cifuentes ME, Shepard AD, Pagano PJ. Nox4 oxidase overexpression specifically decreases endogenous Nox4 mRNA and inhibits angiotensin II-induced adventitial myofibroblast migration. Hypertension 2008; 52: 143-9.

28 Ozumi K, Tasaki H, Takatsu H, Nakata S, Morishita T, Koide S, et al. Extracellular superoxide dismutase overexpression reduces cuff- induced arterial neointimal formation. Atherosclerosis 2005; 181: 55-62.

29 Laflamme K, Roberge CJ, Grenier G, Remy-Zolghadri M, Pouliot S, Baker $\mathrm{K}$, et al. Adventitia contribution in vascular tone: insights from adventitia-derived cells in a tissue-engineered human blood vessel. FASEB J 2006; 20: 1245-7.

30 Kleschyov AL, Muller B, Schott C, Stoclet JC. Role of adventitial nitric oxide in vascular hyporeactivity induced by lipopolysaccharide in rat aorta. Br J Pharmacol 1998; 124: 623-6.

31 Beranova P, Schott C, Chalupsky K, Kleschyov AL, Stoclet JC, Muller B. Role of the adventitia in the cyclic GMP-mediated relaxant effect of $\mathrm{N}$-hydroxy-L-arginine in rat aorta. J Vasc Res 2005; 42: 331-6. 